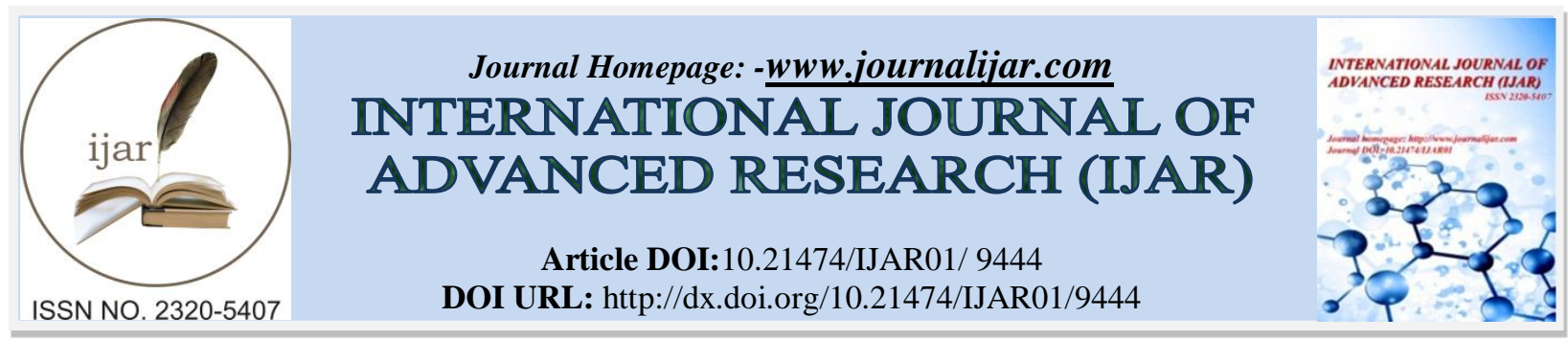

RESEARCH ARTICLE

\title{
PHILOSOPHICAL ANALYSIS FOR MULTI-WORLDS INTERPRETATION OF QUANTUM MECHANICS.
}

School of Physical science and Technology, Guangxi Normal University, Guilin, 541004, P. R. China.

\section{Manuscript Info}

Manuscript History

Received: 24 May 2019

Final Accepted: 26 June 2019

Published: July 2019

\section{Abstract}

The multi-worlds interpretation of quantum mechanics, starting from the realism, opposes the reduction of wave function in Copenhagen interpretation and aims to search for what happens after the measurements and to seek the essence of wave function. Different from the emphasis for consciousness of observers during the measurements in Copenhagen interpretation, the multi-world interpretation inherits the determinism in the aspects of methodology, epistemology, as well as ontology. This paper aims to discuss the concepts of realism and determinism in the multi-worlds interpretation from the viewpoint of methodology, epistemology, as well as ontology and to demonstrate that it transcends the traditional determinism.

Copy Right, IJAR, 2019,. All rights reserved.

\section{Introduction:-}

In physics, the measurement is a far-reaching significant means, by which the physicists seek truth of the physical world. In the classical framework of physics, the measurement intuitively, clearly, and accurately shows the objective world to us, which inherits the philosophical concepts of both the dualism between observers and objective world and the causal determinism. However, based on the mathematical formalism, i.e. matrix mechanics, wave mechanics, and Hilbert space, the birth of quantum theory gives rise to a series of problems in the experiential observations and the measurements, as well as philosophical disputations.

Based on classical physics, the evolution of a quantum system follows two distinct directions. One is the consecutive and reversible evolution without the interference of observers. The other is the inconsecutive and irreversible with quantum jump, due to the interference of the observers. That is to say, it is the interference of the observers, as the subject, that breaks the self-balance and the consecutive and reversible self-evolution of the micro-system, which makes the quantum measurement to be incomprehensible. Such process was called either the "projection postulate", cording to von Neumann [1], or reduction of wave-packet, according to Weiner Heisenberg. The way of interpreting quantum mechanics based on the classical physics pushes the physicists to put forward correspondence principle and principle of complementarity, in order to clarify the confusion, which lays the foundations of division between observers and quantum objects and becomes the opponent focus of other interpretations of quantum theory. Accordingly, the measurement problem became the key problem in both quantum mechanics and scientific philosophy.

In 1957, Hugh Everett, from the cosmological point of view by starting from the core problem, i.e. measurement problem, and combining the quantum and classical systems into one single cosmological system, gave the realistic

Corresponding Author:-J. Wang.

Address:-School of Physical science and Technology, Guangxi Normal University, Guilin, 541004, P. 
and ontological answer and successfully solved the reduction of wave-packet, i.e. the multi-worlds interpretations [2]. The essential difference between the multi-worlds and Copenhagen interpretations lies in [3] "the latter is a philosophic freak, which apriorily builds the real micro-world on the classical physics and denies the reality of micro-world".

\section{The Multi-World Interpretation:-}

According to the multi-worlds interpretation [2, 4], the quantum states in micro-world cannot exist independently. Their existence is relative to the relevant elements outside it, such as the apparatus, the observers, as well as the measuring environment, etc.. Therefore, we should define the quantum states on different branches of the quantum system, according to the state of apparatus, the state of observer, and the state of measuring environment. As a consequence, the state of quantum system embeds in a universal wave function, which is the superposition of wave functions describing all branches of the system.

After the first measurement, the world divides into several parallel-worlds, and the universe falls into multi-verse. After the second measurement, the universe subsequently divides into many parallel branches [4], which can occur everywhere in the universe. Such divisions will continue on with the measurements. We can learn the states of all branches once from the measured results, and each result just occurs in its own branch. Supposing that we use the apparatus " $\mathrm{M}$ " and measure the spin of a quantum system " $\mathrm{S}$ " on the " $\mathrm{x}$ " direction, the original state of " $\mathrm{S}$ " is the eigen-state on " $z$ " direction. Then the original state of the whole universe can be expressed as

$$
\mid \text { original }\rangle_{M} \frac{1}{\sqrt{2}}\left(\left|\downarrow_{X}\right\rangle_{S}+\left|\uparrow_{x}\right\rangle_{S}\right) \text {. }
$$

The state describes the direct product of the states of two systems, i.e. the state of apparatus "M" $\mid$ original $\rangle_{M}$ and the state of the quantum system $\frac{1}{\sqrt{2}}\left(\left|\downarrow_{x}\right\rangle_{S}+\left|\uparrow_{x}\right\rangle_{S}\right)$ before their interactions. Once we have done the measurement, the state function becomes,

$$
\left.\left.\frac{1}{\sqrt{2}}(\mid \text { down }\rangle_{M}\left|\downarrow_{x}\right\rangle_{S}+\mid \text { up }\right\rangle_{M}\left|\uparrow_{x}\right\rangle_{S}\right)
$$

There are two incompatible results, whose superposition appears as the set of multi-systems. The apparatus in each sub-system shows one reading, corresponding to each element in the superposed state [5]. Accordingly, the superposed state divides into two states, which describes, respectively, the observed states in two separated worlds. There are the same quantum system " $\mathrm{S}$ ", same apparatus " $\mathrm{M}$ ", and the same observers in each world. The difference lies in the states of "M" and measured results of "M" observed by the observer, i.e. spin-up in one world and spindown in the other one. So we can get the deterministic fact that each world shows its own observed result, because of the separation after the first measurement. It is the interactions between the apparatus and the system that result in the unitary evolution of the wave function during the processes of quantum measurements, and the measured results can be read in each separated system. Each branch is the real existence in the whole universe, and the observer just locates rightly in each branch. Consequently, the measured result of each branch is the definite reading, which can be considered as the concepts of the classical framework of physics.

In the multi-world interpretation, we need a state vector to describe the state of whole universe, whose evolution follows the linear dynamical wave equation, without the reduction of the wave function [6]. There are many same and parallel real worlds in the whole universe, in which the events and states exist separately and independently. We cannot observe the state of one world in the other parallel ones. In order to completely describe the physical reality, it is requires designating the state vector and dynamical variables of the whole universe. The state vector describing the whole universe divides into the orthogonal vectors, each of which describes the local state of each parallel branch, corresponding to each definite reading of the measurement.

\section{Characteristics of Realism in Multi-Worlds Interpretation:-}

The disputation of quantum mechanics focuses on the measurement problem, which is the core problem of both quantum philosophy and interpretation of quantum theory. The mathematical description for the measurement problem is divided into two parts. Before the measurements, the evolution of the quantum system follows the Schrödinger equation, and the wave functions obey the linear superposition principle and form a state vector space. Once we perform the measurement, the evolution of the system follows another way and collapses into another definite state. The whole process is non-linear, non-deterministic, and dynamically irreversible. 
According to the multi-world interpretation, the fundamental equation of quantum mechanics — the Schrödinger equation, as the fundamental description for the reality, can be applied to describe the whole universe. The state of a quantum system described by the Schrödinger equation represents a reality at a deeper level. In this sense, there is no any difference between the macrocosm and microcosm. Both the apparatus and the observer are described as the forms of quantum mechanics, and thus there is no interference during the process of measurement. The overall measuring system, including the apparatus, the observer, and the quantum object, are described by one single wave function. Accordingly, the measurement is a quantum process, without any special macro-description for the classical apparatus. The wave function of the whole universe evolves according to the Schrödinger equation, which is a deterministic theory. Therefore, the wave function is endowed with the fundamental reality, which gives descriptions to macro-objects (the apparatus), the macro-subject (the observer), and the micro-object (the quantum system).

As one kind of the realism, the realism of quantum state considers the overall measuring system, including the observing subject - the observer, as the quantum reality, which is independent of the observing subject. Therefore, the multi-worlds interpretation insists on the objective realism. During the measurement, the observer can objectively record each result, which corresponds to the state function of each branch. All the branches have the same rank, without any particularity, and thus possess same reality. The emphasis on the relativity of the apparatus, the observer, the quantum system, as well as the measuring environment, and the interpretations of wave function as the fundamental entity demonstrate the wholeness of ontology.

\section{Concepts of Determinism in Multi-Worlds Interpretation:-}

The multi-worlds interpretation describes the state of the whole universe as one entity, i.e. the wave function, whose evolution deterministically follows the Schrödinger equation. It eliminates the problem of reduction of wave-packet and ascribes the collapse to multi-branches of observing states by the observer. Each branch corresponds to the eigen-value of the sub-system. The state function actually doesn't collapse and still satisfies the Schrödinger equation, which is a linear and deterministic process. As long as learning the initial condition of the state function, we can figure out the coming states of the system, which is definitely determinism. Therefore, It admits the deterministic evolution of the microcosm itself in the ontology, which lays solid foundation of determinism.

Whether the self-evolution of microcosm is deterministic depends on its intrinsic properties, which is independent of the observers. However, whether the physicist admits the determinism of the microcosm is a problem of the epistemology. The presupposition for the general validity of quantum mechanics during the evolution of the quantum system believes that the Schrödinger equation is universally applicable to any system and that the deterministically and linearly dynamical equation describes completely and exactly the physical state of the whole universe, which is foundation and starting point of the epistemology. Because of the strict logicality of the mathematics, the multi-worlds interpretation resorts to the mathematical formula, i.e. the Schrödinger equation, and deduces the evolution of the quantum system, which ensures the determinism in the choice of ontology.

Despite the avoidance of reduction of wave-packet and the quantum jump during the evolution, the multi-worlds interpretation [4] doesn't reject the randomness and probability and demonstrates that Born's probability is one of the results of the system of quantum mechanics. It believes that the probability is relative frequency of eigen-values of the observables, which depends objectively on the measuring system overall and represents the probability of the observers' location in each branch. The idea of probability still obeys the causality and believes that the causes of the probability produce the results of probability. Therefore, it insists on the determinism and denies the strict determinism. However, it should be noting that the multi-worlds interpretation doesn't totally negate both the strict determinism and under-determinism [7], which are two kinds of manifestations of the determinism. The strict determinism is considered as one of the special cases and limits of the determinism, while the under-determinism is thought of as one of the phenomena. In terms of the probability of quantum mechanics, the strict determinism is the determinism with probability of " $100 \%$ ", while the under-determinism is the probabilistic determinism, and there is no any contradiction between them. The introduction of probability into the determinism deepens the concept of determinism in the quantum theory, which implies that the determinism of quantum mechanics can be expressed in terms of probability between " 1 " and " 0 " and gives the determinism broader extension.

\section{Summary and Discussion:-}

The multi-worlds interpretation avoids the reduction of wave function by using the division, which removes the dependence of quantum measurement on the observer. Consequently, we needn't suffers from the problem of 
classical world. Both the observers and the quantum system are subjected to the dynamical laws of quantum mechanics. We then describe all scales of the universe as wave functions of quantum mechanics, which includes all the observers and apparatuses. The whole state of the universe is determined by one wave function, which is the superposition of the states of sub-branches, instead of the quantum jump, and never collapses, deterministically evolving according to the Schrödinger equation.

In the theory of multi-world, the quantum mechanics can be considered as an integral, realistic, deterministic, and local theory, which removes the inflexible separation of the ontological reality, seeming more like the classical theory and fitting well with our classical experiences. Therefore, it makes no sense to say that the sub-branch is an isolated system. The observation is not a new process outside the isolated system, but the special case of the interaction intrinsic to the measured quantum system, which establishes the consistence between the mathematical framework of the theory and the observational experience.

The method of description for the universe as one wave function, actually, ontologically sticks to the monism and discards the dualism. The evolutions of all the branches follow the Schrödinger equation, which implies the dualism in the evolutionary pattern of the quantum system. The observers, the apparatus, the quantum system, and the measuring environment are considered as an integrity, which dispels the conflict between the matter and consciousness, the contradiction between the macrocosm and the microcosm, and the division of observing subject and the measured object. Therefore, it transcends the Copenhagen interpretation and moves the way to the holism, the monism, and the determinism.

The evolution of the macro-state of the universe is explained as the division in a local way. However, it is confusing in the interpretations about when the division occurs and the details of the branches. There is no any special interactions and exact definition for the performance of measurement, which gives rise to contradiction between the reversibility of time and the irreversibility of history for the measurement. After decoherence of the sub-branches, they are separated completely, and there is no any interaction between them, which implies the disappearance of the memory. As a result, no any observers can be the testimony of the occurrence of division, which, to some extent, is lost in the agnosticism.

\section{References:-}

1. J. von Neumann, "Mathematical Foundations of Quantum Mechanics", Princeton: Princeton University Press, 1932

2. H. Everett, "The Theory of Universal Wave Function", Ph.D Thesis, Princeton University (1957)

3. H. Everett, "Relative State Formulation of Quantum Mechanics", Rev. Mod. Phys., 29, 454 (1957)

4. B. S. DeWitt and N. Graham, "the Multi-world Interpretation of Quantum Mechanics", Princeton: Princeton University Press, 1973

5. R. N. Graham. "The Measurement of Relative Frequency" In R. Neill Graham, Bryce Seligman DeWitt (Eds.), "the Many-worlds Interpretation of Quantum Mechanics". Princeton, Princeton University Press, 1973

6. J. A. Barrett. "The Quantum Mechanics of Minds and Worlds", New York: Oxford University Press, 1999

7. M. Jammer, "Philosophy of Quantum Mechanics: the Interpretations of Quantum Mechanics in Historical Perspective", John Wiley \& Sons, Inc. 1974. 